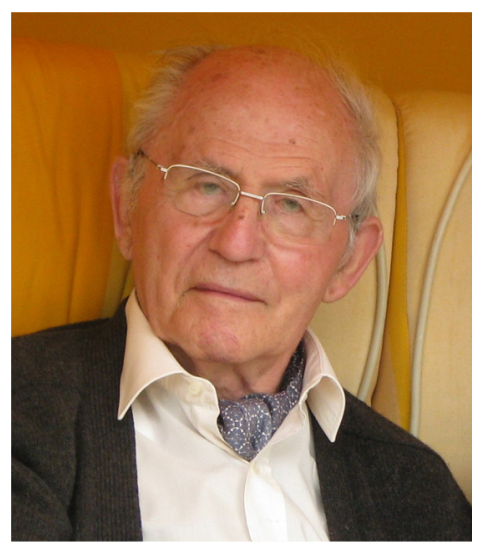

\title{
Hommage au professeur Pierre DUCHÊNE-MARULLAZ (1921-2014)
}

Le professeur Pierre DUCHÊNE-MARULLAZ, professeur de pharmacologie médicale à Clermont-Ferrand, membre correspondant de l'Académie nationale de Médecine, est décédé à Chamalières début juin 2014. Il fut l'un des grands pharmacologues de son temps.

Né en 1921, docteur en médecine en 1946, il fut d'abord formé à la grande physiologie par ses Maîtres, et notamment le professeur Fernand Jourdan, physiologiste à Lyon. Nommé professeur de pharmacologie médicale à la faculté de médecine de Clermont-Ferrand, il dirigea le laboratoire de pharmacologie médicale jusqu'en 1988 et assura un enseignement de pharmacologie passionné et passionnant qui a marqué de nombreuses générations de médecins. Cardiologue, spécialiste de physiologie et pharmacologie cardio-vasculaires, il créa l'unité Inserm U195 et anima une recherche reconnue sur la physiologie, physiopathologie et pharmacologie de la conduction et de l'excitabilité cardiaques, utilisant notamment des modèles animaux originaux, faisant preuve d'une très grand dextérité expérimentale et publiant de 1950 à 1994 près de 300 publications dans les plus grandes revues de physiologie, pharmacologie et cardiologie. Il participa aussi à l'installation hospitalière de la pharmacologie médicale à Clermont-Ferrand, avec en particulier une structure d'exploration électrocardiographique et tensionnelle. Ses élèves furent nombreux tant en faculté de médecine (Jeanine Lavarenne, Alain Eschalier et Claude Dubray, Régine Fabry à Clermont, Philippe Lapalus à Nice, Jean-Pierre Kantelip à Besançon...), qu'en faculté de pharmacie (Alain Combre à Nantes...) ou à l'Inserm (Michel Boucher, Claude Chassaing, Nicole Moins... à l'U195). Il leur a laissé le souvenir d'un pharmacologue très motivé par ses activités de recherche qui savait transmettre sa passion pour une recherche qualifiée aujourd'hui de «translationnelle », qui ne comptait pas son temps, avait initié des premiers travaux de recherche clinique et avait su développer les partenariats public-privé. Très attaché à sa région, il dirigea l'Institut de recherches cardio-vasculaires de Royat, où, en esprit curieux et novateur, il développa, à côté de la recherche fondamentale physiologique et pharmacologique cardio-vasculaire, de 1965 à 1987, les premières études scientifiques, cliniques et épidémiologiques, d'évaluation rigoureuse des pratiques thermales. Ce lieu, connu de beaucoup, fut fréquenté par de jeunes pharmacologues français pour des « vacances d'été » studieuses.

Il fut l'un des fondateurs en 1970 de l'Association des pharmacologistes, ancêtre de la SFPT, où il assura la charge de rédacteur en chef du Journal de Pharmacologie (J Pharmacol [Paris]) durant toute sa période d'édition de 1976 à 1986, date de sa transformation en Fundamental and Clinical Pharmacology.

Homme de grande culture, soucieux des questions humanistes sur la signification de la vie, grand travailleur et illustre défenseur du médicament et d'une pharmacologie systémique reposant sur des bases physiologiques fortes, il laissera le souvenir d'un fondateur d'une grande école hospitalo-universitaire de pharmacologie médicale.

Thérapie adresse ses très sincères condoléances à son épouse et toute sa famille.

Requiescat in pace.

Alain Eschalier (Clermont-Ferrand) Jean-Louis Montastruc (Toulouse) 